\title{
Prediction of Clinical Outcomes of Spinal Muscular Atrophy Using Motion Tracking Data and Elastic Net Regression
}

\author{
David Chen \\ Research Information Solutions \\ and Innovation \\ Nationwide Children's Hospital \\ Columbus, Ohio 43205, USA \\ david.chen3@nationwidechildrens.org \\ Simon Lin \\ Research Information Solutions \\ and Innovation \\ Nationwide Children's Hospital \\ Columbus, Ohio 43205, USA \\ simon.lin@nationwidechildrens.org
}

\author{
Steve Rust \\ Research Information Solutions \\ and Innovation \\ Nationwide Children's Hospital \\ Columbus, Ohio 43205, USA \\ steve.rust@nationwidechildrens.org \\ Leslie Nelson \\ Department of Physical Therapy \\ University of Texas Southwestern \\ Medical Center \\ Dallas, Texas 75235, USA \\ leslie.nelson@utsouthwestern.edu \\ Linda P. Lowes \\ Clinical Therapies Department \\ Nationwide Children's Hospital \\ Columbus, Ohio 43205, USA \\ linda.lowes@nationwidechildrens.org
}

\author{
En-Ju D. Lin \\ Research Information Solutions \\ and Innovation \\ Nationwide Children's Hospital \\ Columbus, Ohio 43205, USA \\ deborah.lin@nationwidechildrens.org \\ Lindsay Alfano \\ Clinical Therapies Department \\ Nationwide Children's Hospital \\ Columbus, Ohio 43205, USA \\ lindsay.alfano@nationwidechildrens.org
}

\begin{abstract}
Spinal muscular atrophy (SMA) is a common muscle disease that can lead to high rate of infant mortality. It is important to be able to quickly and accurately diagnose SMAs as well as track disease progression throughout the treatment process. This study introduced a framework for deriving movement features from motion tracking data, and applied a regularized regression method to predict the gold standard clinical measures for SMA, the CHOP INTEND Extremities Scores (CIES). Our results showed the CIES could be predicted with good accuracy using derived motion features and Elastic Net regression. An RMSE of 8.5 points on CIES was achieved in both cross-validation and prediction on the heldout set. A high ROC-AUC of 0.91 was achieved for discriminating SMA infants from Controls on both session and subject levels. It was concluded that motion tracking devices could potentially be used as a low-cost yet effective method to assess and monitor infants with SMA.
\end{abstract}

\section{CCS CONCEPTS}

- Computing methodologies Supervised learning

- Computing methodologies Feature selection

- Computing methodologies Regularization

Permission to make digital or hard copies of part or all of this work for personal or classroom use is granted without fee provided that copies are not made or distributed for profit or commercial advantage and that copies bear this notice and the full citation on the first page. Copyrights for third-party components of this work must be honored. For all other uses, contact the owner/author(s).

ACM-BCB'18, August 29-September 1, 2018, Washington, DC, USA

(c) 2018 Copyright held by the owner/author(s).

ACM ISBN 978-1-4503-5794-4/18/08.

https://doi.org/10.1145/3233547.3233572

\section{- Computing methodologies $\sim$ Cross-validation \\ - Computing methodologies Motion capture}

\section{KEYWORDS}

Motion tracking, Kinect, Spinal Muscular Atrophy, Regularized Regression, Elastic Net

ACM Reference format:

David Chen, Steve Rust, En-Ju D. Lin, Simon Lin, Leslie Nelson, Lindsay Alfano and Linda P. Lowes. 2018. Prediction of Clinical Outcomes of Spinal Muscular Atrophy Using Motion Tracking Data and Elastic Net Regression. In Proceedings of 9th ACM Int'l Conf. on Bioinformatics, Computational Biology \& Health Informatics (ACM-BCB'18). ACM, NY, NY, USA, 9 pages. https://doi.org/10.1145/3233547.3233572

\section{Introduction}

Spinal muscular atrophy (SMA) type 1 is a rare genetic disease that results in progressive weakness of the skeletal and bulbar muscles. If untreated, it results in death or permanent mechanical ventilation by two years of age. Due to their declining breathing abilities, infants with SMA type 1 are extremely vulnerable to respiratory infection. As the disease progresses, the infants frequently require complex medical care such as supplement tube feeding and suctioning to clear secretions from the airway. This combination of susceptibility to illness and medical equipment needs makes traveling difficult [1, 2]. Recently, the first-ever treatment for SMA type 1 was approved by the FDA and made commercially available. Additionally, there are several promising compounds in the drug development pipeline. 
Traditionally, the Children's Hospital of Philadelphia Infant Test of Neuromuscular Disorders (CHOP- INTEND) is used to quantify the change in motor abilities in these fragile infants [3]. This 17-item assessment looks at the strength of the infant's arms, legs, trunk, and head. The CHOP-INTEND evaluation can be extremely fatiguing to fragile infants [3]. It requires the infant to be placed in taxing positions that put an additional burden on the work of breathing. Administration of the CHOP-INTEND also requires the infant to travel to a regional medical center to see a trained evaluator familiar with this rare disease. Therefore, an evaluation system that could track the infants' natural movements in their homes would be a significant addition to the medical field.

Motion tracking devices such as Microsoft Kinect are potentially useful for quickly and economically collecting data without fatiguing the infant or requiring the family to travel. To this end, a pilot study [4] looked at the initial feasibility of using Kinect data for capturing the arm and leg movements of patients. The positional data obtained from the Kinect were analyzed using Convolutional Neural Networks and showed promise for disease progression [5]. Although there has been some research into the utility of using a stereo-optic camera such as the Kinect for medical applications, it is still under-discovered. The ability to remotely capture and characterize infant motion using automatically extracted motion features such as distance, direction, direction change, velocity, and acceleration, from an inexpensive motion tracking device could revolutionize infant assessments for SMA. Once established, such a framework can facilitate the development of evaluation tools for other more common, yet difficult to diagnose, conditions such as cerebral palsy at the level of ease and low cost that were not previously available [6].

Elastic net (EN) regularized regression has gained popularity in recent clinical studies including genetic research [7-9]. EN was first developed by Zou and Hastie to overcome some of the drawbacks of two predecessor algorithms: Lasso and Ridge [10, 11]. The penalty parameter $\alpha$ determines the weight that is given to an EN model during the regularization process; as a result, the resulted model can be flexibly tuned to behave similarly to either the lasso or the ridge, or any percentage of the mix of the two. The EN extends and inherits the merits of both lasso and ridge, but removes any negative effects caused by high correlations in the data $[9,12]$ However, to the best of our knowledge, there is no previous work on applying and evaluating the EN approach to assess SMA, especially in the context of using motion tracking data.

The main contributions of this paper are summarized as follows:

- A new feature engineering framework was developed for quantitatively characterizing the extremity (limb) movement of subjects. Although this paper involved patients with SMA, the feature engineering framework can be applied to any research using motion tracking data. (Section 3.2)

- Elastic net regularized regression models were implemented for CHOP INTEND Extremities Score (CIES) prediction using motion tracking data and their performance was compared, and evaluated. (Section 3.3)
- CIES predictions were further used to discriminate SMAs from Controls on both session and subject levels to demonstrate the utility of using predicted CIES. (Section 3.4)

\section{Motion Tracking Data and CHOP INTEND Extremities Scores}

Tracking data were collected from infants under 180 days of age using the Microsoft Kinect V2 camera system. 61 subjects including 34 subjects with SMA (SMA thereafter) and 27 normal controls ("Controls" thereafter) were recruited by two authors (LL and LA) at Nationwide Children's Hospital Muscular Dystrophy Association Clinic (NCH MDAC) and by one author (LN) at the University of Texas Southwestern Medical Center (UTSW MC). The average age of the infants with SMA was 401 days (range 121590). As expected, the control group was much younger (Mean 85; range 17-185) because the infant can no longer be tested after they start to roll or crawl as he/she will not stay under the camera. Untreated infants with SMA type 1 never roll and those treated infants in this study did not do so until they were significantly older.

The study and data collection was approved by the institutional review board. All three of the authors collecting the data participated in in-person training and reviewed a detailed instruction manual on ACTIVE mini procedures. These individuals all have extensive experience with the CHOP-INTEND and serve as international instructors on the assessment procedures. Interrater reliability between the 3 authors was established quarterly via web based video reviews. Informed consents were obtained from the parents of all infants who participated in this study.

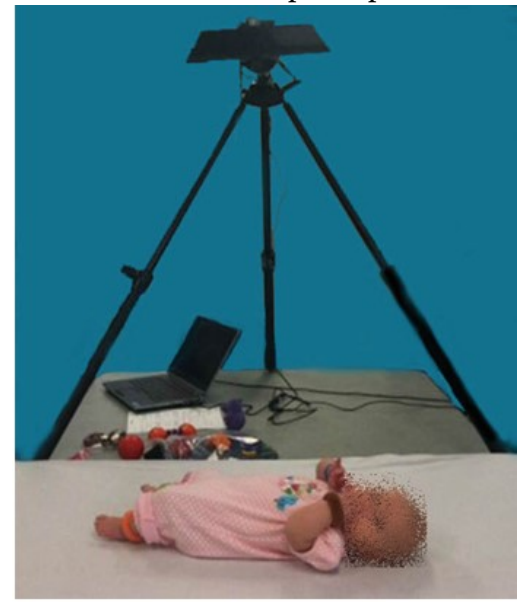

Figure 1: Camera Configuration for Collecting Motion Tracking Data

All subjects had multiple visits, and at each visit, most subjects had two video recording sessions. Each recording session was set up as shown in Fig. 1. During each session, Kinect tracked the 3D motion of the four limbs of subjects with each limb clearly marked with a different colored latex-free elastic bandage on the hand or foot, which are called tracking locations. 


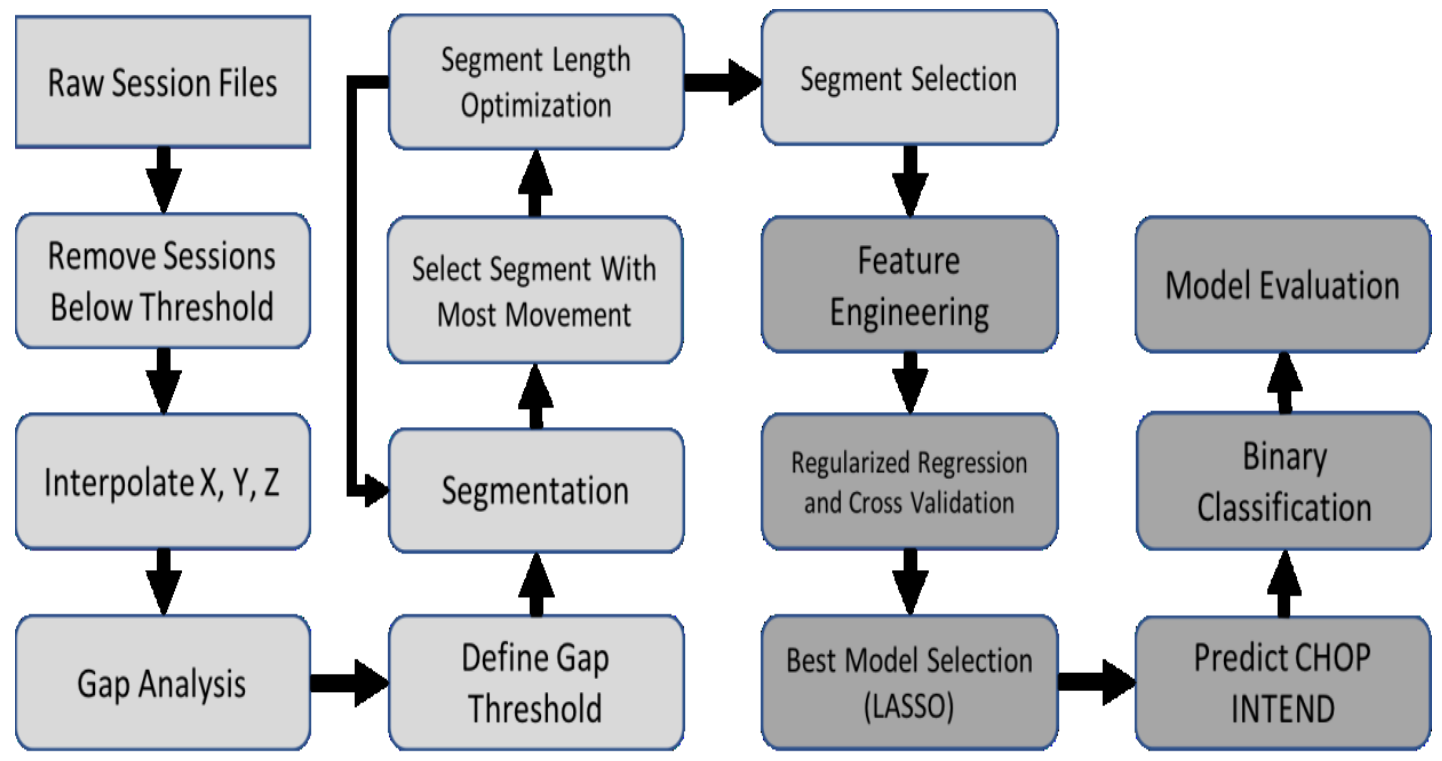

Figure 2 Data Processing and Predictive Modeling Workflow

The camera was positioned directly above the infant. Each session resulted in a separate Session Tracking File (STF) with five columns of information encoded: tracking time (in seconds), tracking location (one of the four limbs), and $\mathrm{x}, \mathrm{y}, \mathrm{z}$ positions (in $\mathrm{mm})$. In the end, we obtained 678 STFs from 61 subjects for subsequent analysis.

The CIES was administered at every data collection session. Each new visit was subject to a new evaluation, but different trials during the same visit were given the same score.

\section{Methods}

The overall data analysis workflow is shown in Fig. 2. Light background boxes are data preprocessing tasks and dark background boxes are predictive analytics tasks. The next few sections provide the details of each task in the workflow.

\subsection{Data Preprocessing}

STFs are different in length. According to our neuromuscular clinicians, a session that has a total length of 30 s or less is considered to be clinically insignificant for patient evaluation. Therefore, during preprocessing, we removed all sessions that were shorter than 30 s. Also, Kinect tracks $\mathrm{x}, \mathrm{y}, \mathrm{z}$ locations at up to $30 \mathrm{~Hz}$; however, recordings were not always at the maximum rate causing missing data in $\mathrm{x}, \mathrm{y}, \mathrm{z}$ positions. To allow correct motion feature calculation, we filled all missing values in $\mathrm{x}, \mathrm{y}, \mathrm{z}$ positions using linear interpolation. We implemented the interpolation function using the popular Zoo library in R [13]. The average percentage of data points that require interpolation from a file was less than $5 \%$.

Time gaps between two consecutive tracking points can affect the accuracy of motion feature calculation. In our case, a gap is defined as any two adjacent tracking points that are more than $0.033 \mathrm{~s}$ apart $(1 / 30 \mathrm{~Hz})$. According to our neuromuscular clinicians, smaller (2s or shorter) and a small number of gaps (1 or fewer) are generally acceptable. Based on our initial gap analysis, a gap threshold of $2 \mathrm{~s}$ was found to be appropriate for identifying gaps in the sessions. With 2 s gap threshold, $93 \%$ of the files were found to have no gaps with only $1 \%$ of the files having more than 1 gap. Once the gap threshold was defined (2s in our case), the next step was to divide the whole session into segments based on the location of gaps in each session. For example, if a session has only one gap, two segments will result from that session.

After segmentation, we obtain 1 or more segments from each session. To narrow it down to only one segment per session, we selected the segment with the Longest Total Excursion (LTE) as the most representative segment based on the magnitude of the total 3D movement of all four limbs. Then, a moving window method was used to find the best same-length sub-segment for each session. These experiments aimed to derive segments of the same length for subsequent analysis so that the variation in segment length, which could have negatively affected model accuracy and interpretation, could be eliminated. We experimented with different moving window lengths: 30s, 60s, and $90 \mathrm{~s}$. The rule to choose the best final moving window segment was also LTE.

The result of the data preprocessing step was a list of the same length (90s for example) segments, each representing the corresponding session the segment was extracted from.

\subsection{Feature Engineering}

The feature engineering process was to generate the features for machine learning based on the laws of motion physics. In the following sections we first discuss the way we developed motion features and then discuss how we converted motion features into features that can enter a regularized regression model. 
Table 1: Motion Feature Categories and Motion Features for Characterizing Movement

\begin{tabular}{|c|c|c|}
\hline $\begin{array}{l}\text { Motion Featur, } \\
\text { Category }\end{array}$ & Motion Feature & Description \\
\hline Distance & $\begin{array}{l}3 \times 1 \mathrm{D} \text { distance } \\
3 \times 2 \mathrm{D} \text { distance } \\
1 \times 3 \mathrm{D} \text { distance }\end{array}$ & $\begin{array}{l}\text { total excursion in } 1 \mathrm{D} \\
\text { total excursion in } 2 \mathrm{D} \\
\text { total excursion in } 3 \mathrm{D}\end{array}$ \\
\hline Direction & $\begin{array}{l}3 \times 2 \mathrm{D} \text { direction } \\
3 \times \sin (2 \mathrm{D} \\
\text { direction) } \\
3 \times \cos (2 \mathrm{D} \\
\text { direction) }\end{array}$ & $\begin{array}{l}\text { direction between pairs of } \\
\text { the axis in radian } \\
\text { sine transformation of } 2 \mathrm{D} \\
\text { direction } \\
\text { cosine transformation of } \\
\text { 2D direction }\end{array}$ \\
\hline $\begin{array}{l}\text { Direction } \\
\text { Change }\end{array}$ & $\begin{array}{l}3 \times 2 \mathrm{D} \text { direction } \\
\text { change } \\
3 \times \sin (2 \mathrm{D} \\
\text { direction change) } \\
3 \times \cos (2 \mathrm{D} \\
\text { direction change })\end{array}$ & $\begin{array}{l}\text { direction change between } \\
\text { pairs of axis } \\
\text { sine transformation of } \\
\text { direction change } \\
\text { cosine transformation of } \\
\text { direction change }\end{array}$ \\
\hline Velocity & $\begin{array}{l}3 \times 1 \mathrm{D} \text { speed } \\
3 \times 2 \mathrm{D} \text { speed } \\
1 \times 3 \mathrm{D} \text { speed }\end{array}$ & $\begin{array}{l}\text { average speed in } 1 \mathrm{D} \\
\text { average speed in } 2 \mathrm{D} \\
\text { average speed in } 3 \mathrm{D}\end{array}$ \\
\hline Acceleration & $\begin{array}{l}3 \times 1 \mathrm{D} \text { acceleration } \\
3 \times 2 \mathrm{D} \text { acceleration } \\
1 \times 1 \mathrm{D} \text { acceleration }\end{array}$ & $\begin{array}{l}\text { rate of velocity change in } \\
1 \mathrm{D} \\
\text { rate of velocity change in } \\
2 \mathrm{D} \\
\text { rate of velocity change in } \\
3 \mathrm{D}\end{array}$ \\
\hline
\end{tabular}

3.2.1 Motion Feature Categories and Motion Features. We characterized the individual movement of each one of the four limbs in five Motion Feature Categories (MFCs): distance, direction, direction change, velocity, and acceleration. Based on motion physics properties we believe these five categories are sufficient to characterize the differences in a subject's ability to move. Within each MFC, we further developed individual Motion Features (MFs) to characterize the granular movement within each category in one $(x, y, z)$, two $(x y, x z, y z)$ and three dimensions (xyz) wherever applicable.

As shown in Table 1, for example, the distance MFC contains 7 individual distance features: distances in 1 dimension along $\mathrm{x}$, $\mathrm{y}, \mathrm{z}$ respectively, distances in 2 dimensions along $\mathrm{xy}, \mathrm{xz}, \mathrm{yz}$ respectively, and distance in 3 dimensions along xyz. Velocity and acceleration MFCs both contain 7 individual features and were calculated similar to the distance MFC. For direction and direction change features, both their sine and cosine transformations along with original radian values were calculated. All MFs were calculated using standard physics movement formulas in [14] for each time point in the session (or equivalently segment). The value will be NULL if an MF value does not exist, for example, the first value of the direction feature.

We calculated all 39 MFs in $R$ at each time point for all session segments.

3.2.2 Converting Motion Features to Machine Learning Features Using K-means Clustering and Histogram Binning. So far, the quantitative characterization is at the time point level. However, to enable machine learning on the session level, we needed to aggregate MFs on the time point level to Machine Learning Features (MLFs) on the session level (i.e., segment level) so that all sessions would have the same set of features during machine learning. The MLFs we developed were Histogram Features (HFs), which are the percentage of time each k-means cluster, executed for each MFC, occurs in each session. Fig. 3 illustrates the detailed process.

In addition to what is described in Fig. 3, we also experimented as follows:

- All clusters are global clusters as the cluster centers were generated by using all time points from all sessions.

- To find the optimal number of clusters during k-means for each MFC, we implemented the Elbow approach [15], which often serves as a useful heuristic for finding the optimal number of clusters. Based on our experiments of $10,20, \ldots 100$ clusters, we found 30 clusters as an appropriate final number to use.

- We also experimented to find whether prediction results are sensitive to different $\mathrm{k}$-means settings. We experimented with different number of iterations for k-means (1, 10 and 100), three k-means algorithms: Lloyd/Forgy [16], HartiganWong [17], MacQueen [18], and different number of initial random centers $(1,5,10)$.

\subsection{CHOP INTEND Extremities Scores Prediction and Evaluation}

To establish the validity of the ACTIVE mini we selected the 9 items on the original CHOP-INTEND that assessed arm or leg movements as our gold standard. We summed the score for both the right and left side on these items and refer to this score as the CIES (CHOP INTEND Extremities Scores). We specifically choose only the 9 extremity items and not the head and trunk items as ACIVE mini evaluates the spontaneous movement of all 4 extremities. Currently, head and trunk movements are not captured with ACTIVE mini. We hypothesized that for the initial analysis using only items with a direct comparison to ACTIVE mini would allow more accurate modeling by not introducing features not directly collected with ACTIVE mini. CHOPINTEND items are scores on a 4-point scale, therefore, scores on the CIES could range from 0 to 72 with 0 representing a very low motor function and 72 representing a high motor function. 


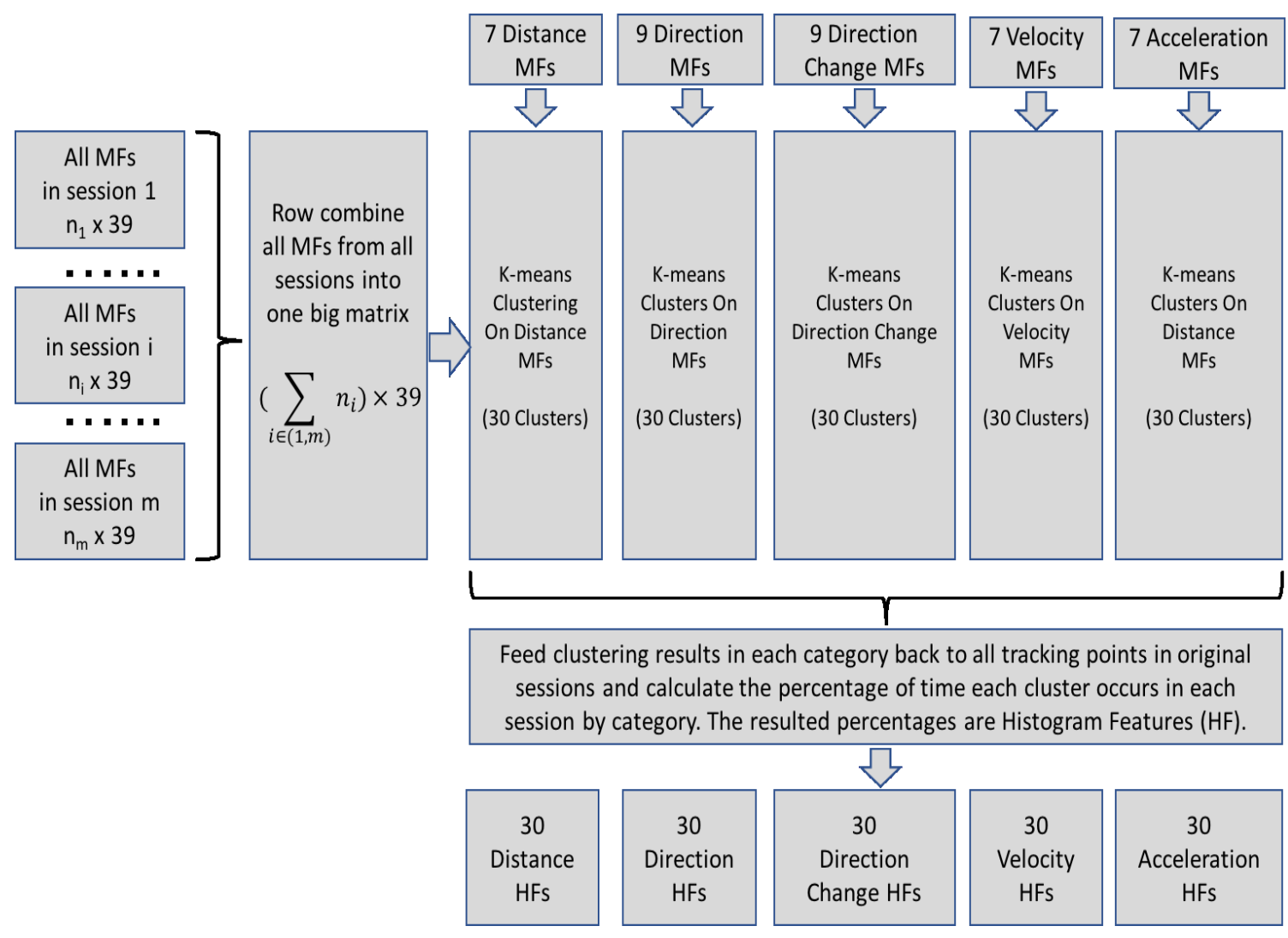

Figure 3: Feature Generation Using K-means Clustering and Histogram Binning

The Elastic Net (EN) algorithm [10] has been implemented in the Glmnet package, a robust and fast R solution [19]. Glmnet formulates $\mathrm{EN}$ as the following objective function:

$$
\min _{\beta_{0} \beta} \frac{1}{N} \sum_{i=1}^{N} w_{i} l\left(y_{i}, \beta_{0}+\beta^{T} x_{i}\right)+\lambda\left[(1-\alpha) \mid \frac{|\beta| \|_{2}^{2}}{2}+\alpha\|\beta\| \|_{1}\right](1)
$$

where $\alpha$ is the elastic net penalty that controls the behavior of the model as either lasso $(\alpha=1)$ or ridge $(\alpha=0)$ or any percentage of mix of the two. Parameter $\lambda$ controls the strength of the penalty. Details of the EN implementation in Glmnet and meanings of other symbols in the above equation can be found in $\mathrm{R}[10]$.

Different settings of $\alpha$ and $\lambda$ were experimented with for finding the best model. $\alpha$ was set to increase from 0 to 1 with 0.1 point incremental. Therefore, $11 \mathrm{EN}$ models resulted. Once $\alpha$ was decided, $\lambda$ was free to take and we experimented with a full range of valid $\lambda$ values given $\alpha$.

We evaluated the implications of variable selection on model performance given different $\alpha$ and $\lambda$ settings by visualizing the coefficient paths of different models. The Root Mean Squared Error (RMSE) between observed and predicted CIES was calculated to compare all 11 models.

We divided all data into $75 \%$ for training and $25 \%$ for testing. Using the training data, we implemented a Cross-Validation (CV) approach to choose the best regularization model with the smallest RMSE. 10 randomized folds were generated for each CV run. Further, we compared the RMSE during CV with that on the test set. Finally, we applied the best model to the whole dataset to generate the predicted CIES for all sessions.

\subsection{Subject Type Prediction and Evaluation}

Given the predicted CIES from the previous step, we produced the binary labels (SMA vs. Control) on both session and subject levels. Binary prediction not only helped with diagnosis of SMA but also allowed us to decide an operating point that was not previously available for discriminating SMAs from Controls.

CIES prediction was performed initially on the session level; however, a subject could have more than one session for the same visit. For this, we experimented with different ways of aggregating the session level results to the subject level. Average prediction was calculated based on 1 visit, 2 visits and up to the maximum number of visits for all subjects. We calculated AUCs for all experiments and decided on the sensitivity of binary classification to different session aggregation methods.

Finally, we examined how well predicted values align with observed values on an individual basis. The progression plots are reported here for the three selected SMA subjects that had the highest number of visits. The plots illustrate the utility of using the predicted scores to track the progression of treated patients with SMA. 
Table 2: Summary of Lasso Coefficients by Motion Feature Category (MFC)

\begin{tabular}{|c|c|c|c|}
\hline MFC (A) & $\begin{array}{c}\text { Number of } \\
\text { Variables } \\
\text { in Lasso With } \\
\text { Minimum } \\
\text { Error (B) }\end{array}$ & $\begin{array}{c}\text { Average } \\
\text { Absolute Value } \\
\text { of Coefficients } \\
\text { in Lasso (C) }\end{array}$ & $\begin{array}{c}\text { MFC } \\
\text { Influence } \\
\text { Indicator } \\
\text { (D) }\end{array}$ \\
\hline $\begin{array}{c}\text { Direction } \\
\text { Change }\end{array}$ & 13 & 0.71 & 9.23 \\
\hline Distance & 8 & 0.92 & 7.36 \\
\hline Acceleration & 9 & 0.44 & 3.96 \\
\hline Direction & 1 & 1.31 & 1.31 \\
\hline Velocity & 3 & 0.32 & 0.96 \\
\hline
\end{tabular}

\section{Results}

During data preprocessing, a 2s gap threshold was found to be appropriate for segmenting the sessions. As a result, 93\% of the files were found to have no gaps and only $1 \%$ found to have more than 1 gap. In deciding the segment length threshold, 90s was found to be the best number (among 30s, 60s, and 90s) to ensure best modeling results based on a moving window method of retaining the best representative segment. After data preprocessing, we retained 581 sessions (i.e. 581 best 90 s segments, one for each session) for regularized regression modeling.

During k-means, we found the CIES prediction accuracy was not sensitive to the number of clusters as long as the number was 30 or more. The prediction results were also not sensitive to the choice of k-means algorithm, number of initial random centers and number of iterations for at least 10 or more. The difference in RMSE was within 0.5 point for these different experiments.

The Lasso model significantly reduced the magnitude of the model coefficients and resulted in a much smaller number of variables, 34 features, selected from the original 150 features space. As shown in Table 2, the final Lasso model included 9 acceleration features, 8 distance features, 1 direction feature, 13 direction change features, and 3 velocity features. The MFC Influence Indicator was calculated as the product of column B and column $\mathrm{C}$ in Table 2. It quantified the influence of each feature category on the regression result with a larger number suggesting higher influence.

Cross-validation on all 11 regularized models using the training data (75\% of all data) produced RMSEs ranging from 8.55 to 10.08 with Lasso being the best performer, Ridge being the worst and Elastic net (different percentages of mixing Lasso and Ridge) in the middle. However, most Elastic net models performed very closely to the Lasso by within 0.5 point difference, small enough to be considered clinically significant. The reason we finally chose the Lasso model was simply based on a preference of model simplicity. The RMSE on the held-out data using the Lasso model was also small at 8.58.

The best Lasso model based on RMSE during CV was then applied to the entire dataset to produce the predicted CIES for all sessions. The scatter plot in Fig. 4 shows the good linear relationship between the predicted values and the observed values. The RMSE on predicting the entire data set was 8.62.

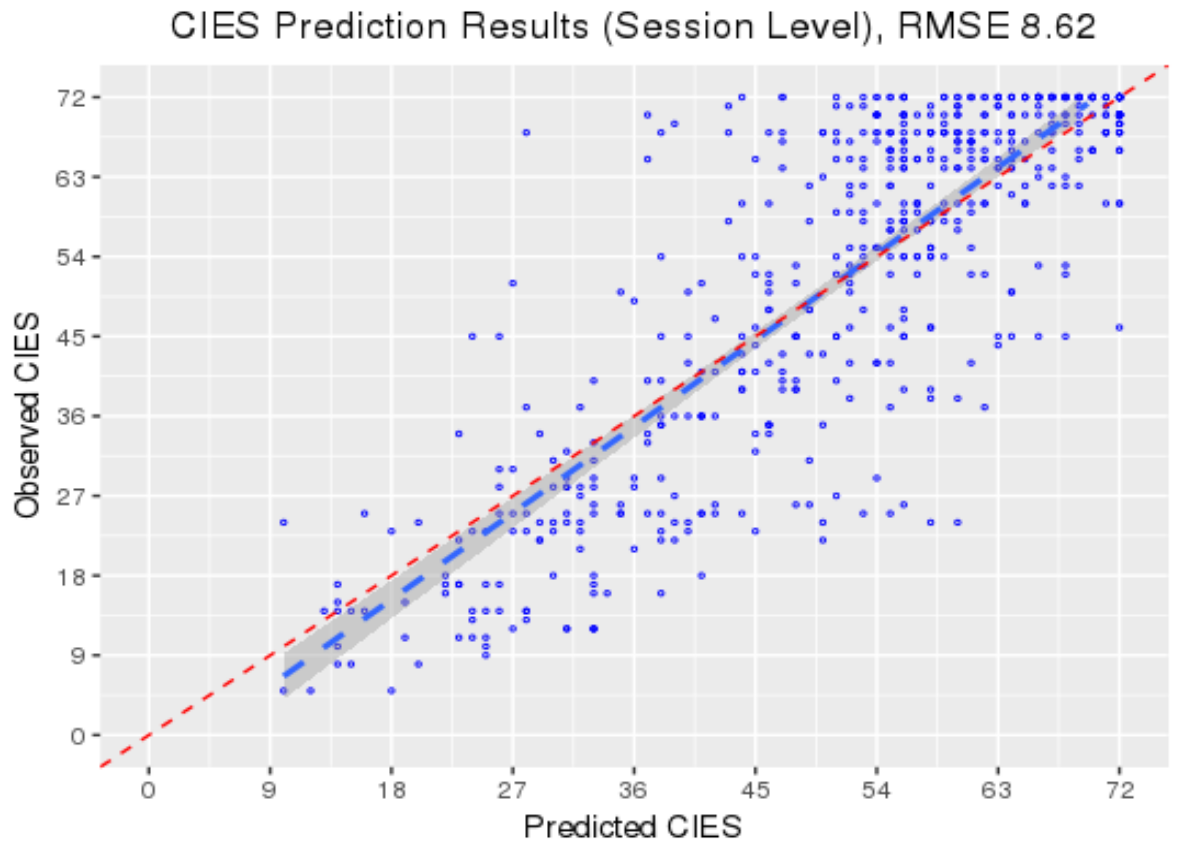

Figure 4: CIES Prediction Accuracy: Predicted vs Observed 

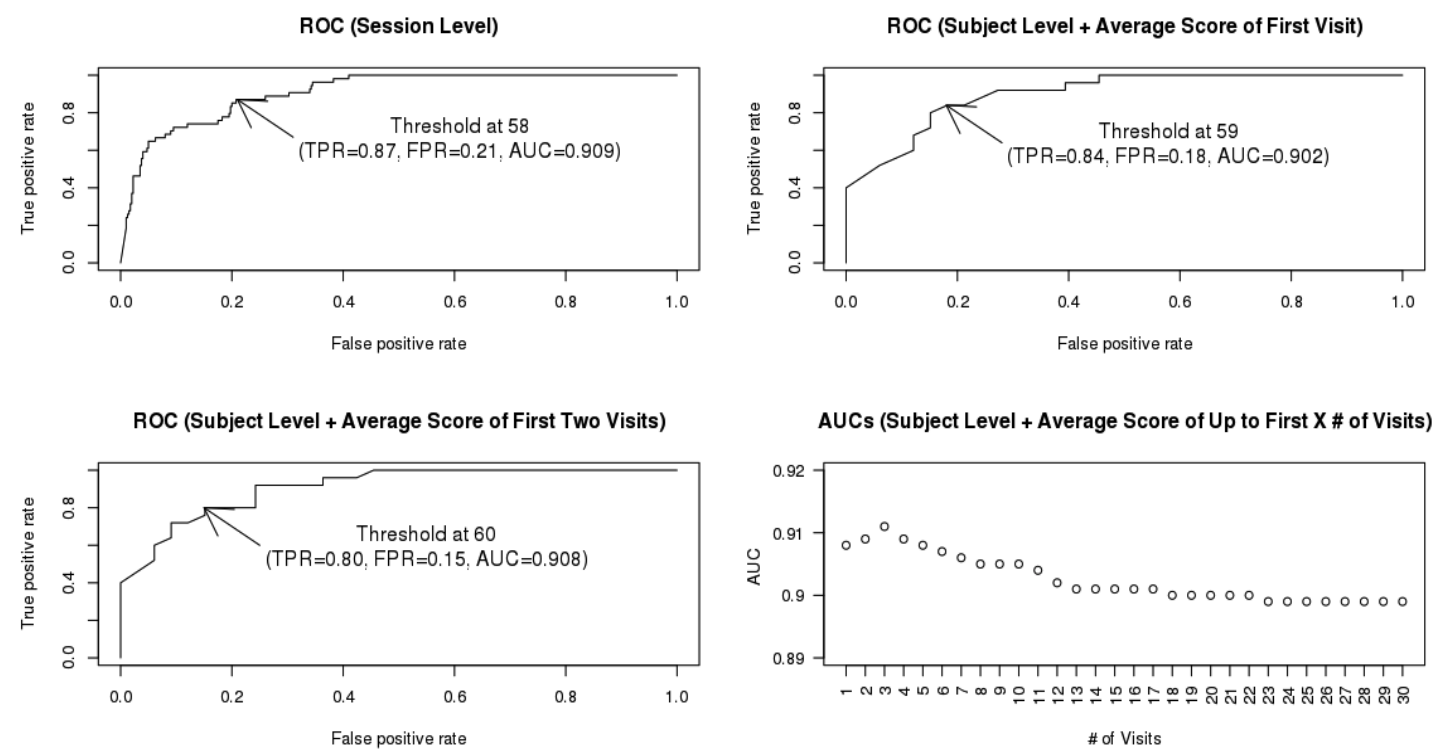

Figure 5: ROC, AUC and Operating Points on both Session and Subject Levels
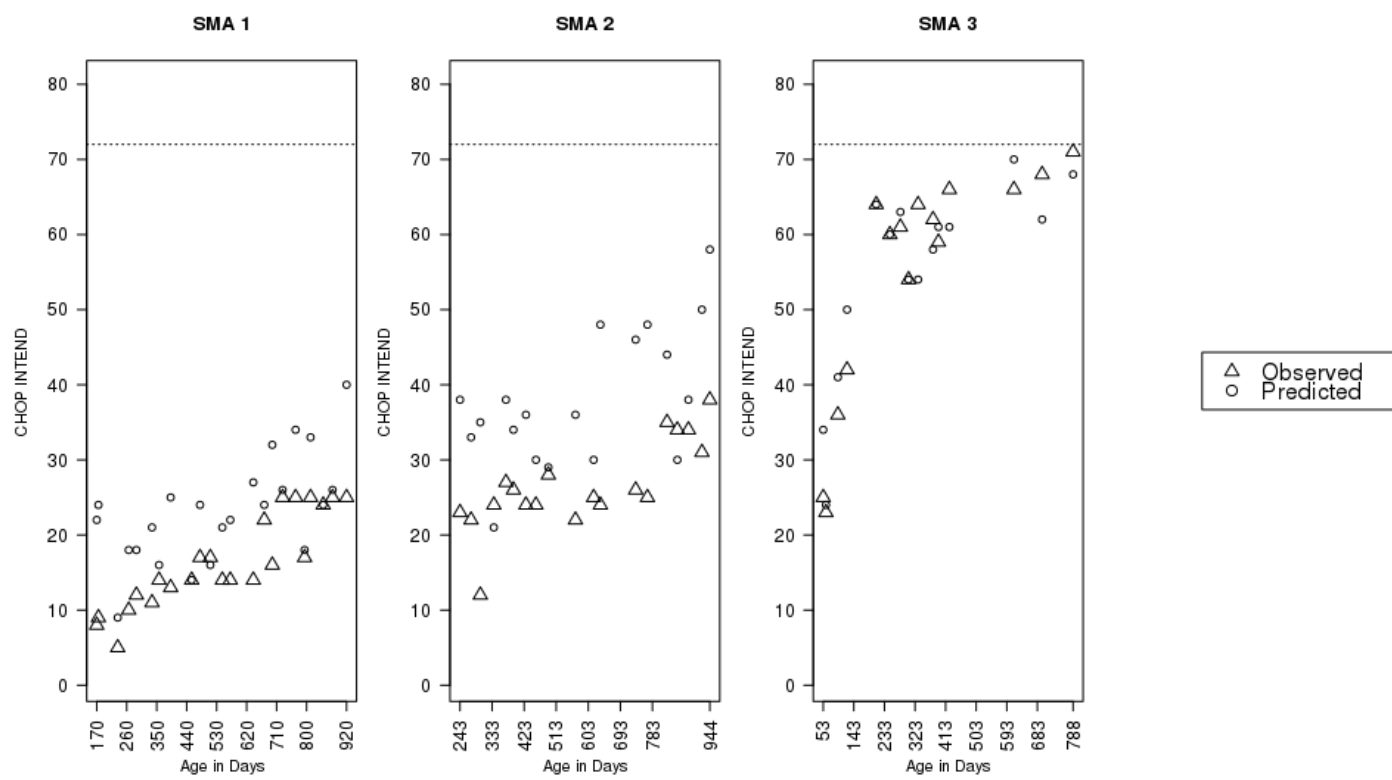

Figure 6: Progression plot of three selected patients with SMA undergoing treatment.

Finally, we calculated the AUCs based on binary label prediction using the predicted CIES at both session and subject levels (Fig. 5). We also calculated the optimal cut-off points that best balanced a high true positive pate (TPR) and a low false positive rate (FPR). The operating points on both session and subject levels were around 59.

\section{Discussion}

The motion features we developed were found to be very effective predictors given both low RMSE and high AUCs. It was further discovered that direction change and distance MFC have both more features in the final model and relatively larger absolute coefficients suggesting their higher influence on the outcome than other MFCs. The most influential MFC was found to be the direction change, followed by distance, acceleration, direction and velocity. These machine-learned features correspond well with the clinical knowledge that the movements of highly functional patients are usually longer and more sophisticated.

In our case, Lasso greatly simplified the final model by achieving $80 \%$ reduction of the variable space while still maintaining high prediction accuracy. This observation is consistent with previous studies using Lasso and medical data [9, 20]. As shown in Fig. 4, the errors of Lasso prediction on the whole 
set were evenly distributed on both sides of the 45-degree line (reference line) with the fitted line being very close to the reference suggesting good model fit. The confidence interval of the fitted line (rendered as the grey bounding box) was tight suggesting good overall agreement between observed values and predicted values.

Our predicted CIES were in good alignment with the observed scores and the underlying clinical conditions of the selected subjects as shown in Fig. 4-6. Using the predicted scores to identify patients with SMA vs. non-SMA controls, the TPR was consistently over $80 \%$, and FPR consistently below $21 \%$ using optimal operating points 58, 59 and 60 in each case respectively (optimization in terms of good balance between high TPR and low FPR). The AUCs (ranging from 0.89 to 0.91 ) were also found consistently high given different ways of aggregating session level results. The SMA progression plots for selected subjects in Fig. 6 showed good agreement between observed and predicted CIES over time. Our results verified the utility of Lasso in predicting clinical outcomes using motion tracking data.

In summary, our study is among the first to implement a regularized approach to predict and track SMA patients using motion tracking data. Although conducted in a clinical setting, the feature engineering and regularized regression approach we proposed could potentially be applied in any other setting using motion tracking data.

\section{Limitations}

Our study may be subject to the following limitations. First, in kmeans clustering, we found 30 clusters as the threshold to result in a right balance between clustering performance and prediction accuracy. However, this number may change given different sets of experimental data. Also, if the lowest prediction error is the primary goal, it may be more practically preferred to start with a bigger number of $\mathrm{k}$ and leverage regularization process to optimize the final variable space. Second, k-means and histogram binning together served as the only method we attempted to engineer motion features for EN regression. It is likely that other vectorization methods may work equally well, if not better, in CIES prediction such as distribution measures of the primitive motion features (i.e., mean, median, standard deviation, quantile, etc.). Due to scope and time constraints, we have not experimented with all these possible feature configurations. Finally, although our analysis resulted in direction change as the most influential feature category, this result is subject to interpretation given a specific model implementation. This ranking may be sensitive to different settings of the input data, and the number of clusters configured in the k-means clustering.

\section{Conclusions}

This paper details a feature engineering framework to predict SMA clinical outcomes using motion tracking data that achieved high accuracy of prediction results using regularized regression. Our findings have implications in applying such predictive systems to reduce the high cost of traditional clinical methods of quantifying movement ability in infants with SMA. For potential future work it would be interesting to explore the possibilities of extending our framework to predict outcomes in other clinical and non-clinical settings that utilize motion tracking data.

\section{Acknowledgement}

The authors would like to thank Dr. Yungui Huang for supervision of R\&D activities, Jesse Haines for project management, Jeremy Patterson for insights on using motion tracking devices, Maggie Dugan for data collection and preparation, and Madeleine Schroeder for assistance in editing.

\section{References}

[1] Iannaccone, S. T., Smith, S. A. and Simard, L. R. Spinal muscular atrophy. Curr Neurol Neurosci Rep, 4, 1 (Jan 2004), 74-80.

[2] Lefebvre, S., Burglen, L Reboullet, S, Clermont, O, Burlet, $\mathrm{P}$, Viollet, L, Benichou, B., Cruaud, C., Millasseau, P., Zeviani, M. and et al. Identification and characterization of a spinal muscular atrophy-determining gene. Cell, 80, 1 (Jan 13 1995), 155-165.

[3] Glanzman, A. M., Mazzone, E., Main, M., Pelliccioni, M., Wood, J., Swoboda, K. J., Scott, C., Pane, M., Messina, S., Bertini, E., Mercuri, E. and Finkel, R. S. The Children's Hospital of Philadelphia Infant Test of Neuromuscular Disorders (CHOP INTEND): Test development and reliability. Neuromuscular Disord, 20, 3 (Mar 2010), 155-161

[4] Lowes, L. P., Alfano, L. N., Yetter, B. A., Worthen-Chaudhari, L., Hinchman, W., Savage, J., Samona, P., Flanigan, K. M. and Mendell, J. R. Proof of concept of the ability of the kinect to quantify upper extremity function in dystrophinopathy. PLoS currents, 5 (2013).

[5] Soran, B., Lowes, L. and Steele, K. M. Evaluation of Infants with Spinal Muscular Atrophy Type-I Using Convolutional Neural Networks. Lect Notes Comput Sc, 9914 (2016), 495-507.

[6] Little, S. E., Janakiraman, V., Kaimal, A., Musci, T., Ecker, J. and Caughey, A. B. The cost-effectiveness of prenatal screening for spinal muscular atrophy. American journal of obstetrics and gynecology, 202, 253 (Mar 2010), e1-7.

[7] Guo, Y. The Noncompacted Folding of Proteins by Modified Elastic Net Algorithm. J Comput Biol, 22, 7 (Jul 2015), 609-618.

[8] Hughey, J. J. and Butte, A. J. Robust meta-analysis of gene expression using the elastic net. Nucleic Acids Res, 43, 12 (Jul 13 2015).

[9] Waldmann, P., Meszaros, G., Gredler, B., Fuerst, C. and Solkner, J. Evaluation of the lasso and the elastic net in genome-wide association studies Front Genet, 5 (Oct 1 2014).

[10] Zou, H. and Hastie, T. Regularization and variable selection via the elastic net (vol B 67, pg 301, 2005). J R Stat Soc B, 67 (2005), 768-768.

[11] $\mathrm{Ng}, \mathrm{A}$. Y. Feature selection, L1 vs. L2 regularization, and rotational invariance Proceedings of the twenty-first international conference on Machine learning (2004), 78.

[12] Witten, D. M., Shojaie, A. and Zhang, F. The Cluster Elastic Net for HighDimensional Regression With Unknown Variable Grouping. Technometrics, 56, 1 (Feb 20 2014), 112-122.

[13] Achim Zeileis, Gabor Grothendieck, Jeffrey A. Ryan, Joshua M. Ulrich and Andrews, F. Zoo Library for R. City, 2016.

[14] Young, H. D., Freedman, R. A. and Ford, L. University Physics With Modern Physics (Chapter 3). Pearson Education, San Francisco, CA, 2016.

[15] Kodinariya, T. M. and Makwana, P. R. Review on determining number of Cluster in K-Means Clustering. International Journal of Advance Research in Computer Science and Management Studies, 1, 6 (2013), 90-95

[16] Lloyd, S. P. Least-Squares Quantization in Pcm. Ieee T Inform Theory, 28, 2 (1982), 129-137.

[17] Hartigan, J. A. and Wong, M. A. Algorithm AS 136: A k-means clustering algorithm. Journal of the Royal Statistical Society. Series C (Applied Statistics), 28,1 (1979), 100-108

[18] MacQueen, J. Some methods for classification and analysis of multivariate observations. Proceedings of the fifth Berkeley symposium on mathematical statistics and probability, 1, 14 (1967), 281-297.

[19] Hastie, T. and Qian, J. R-package glmnet: Lasso and elastic-net regularized generalized linear models., City, 2014.

[20] Li, Z. and Sillanpaa, M. J. Overview of LASSO-related penalized regression methods for quantitative trait mapping and genomic selection. Theor Appl Genet, 125, 3 (Aug 2012), 419-435. 